\title{
INVESTIGATION OF DIFFERENT HYDROGELS FOR NUCLEUS REPLACEMENT - A BIOMECHANICAL STUDY
}

\author{
Sandra Reitmaier (1), Uwe Wolfram (1), Anita Ignatius (1), Hans-Joachim Wilke (1), \\ José M Martín-Martínez (2), Joana Silva-Correia $(3,4)$, Joaquim Miguel Oliveira $(3,4)$, \\ Rui Luís Reis $(3,4)$, Hendrik Schmidt (1) \\ 1. Institute of Orthopaedic Research and Biomechanics, University of UIm, Germany; \\ 2. Adhesion and Adhesives Laboratory, University of Alicante, Spain; \\ 3. 3B's Research Group, Univ. Minho, Portugal; \\ 4. ICVS/3B's - PT Government Associate Laboratory, Braga/Guimarães, Portugal
}

\section{Introduction}

Hydrogels are considered promising for disc regeneration strategies. However, it is currently unknown whether the destruction of the natural interface between nucleus and surrounding structures caused by nucleotomy and an inadequate annulus closure diminishes the mechanical competence of the disc.

To clarify these mechanisms and to evaluate whether hydrogels are able to restore the biomechanical behaviour of the disc a combined in vivo and in vitro and approach was used.

\section{Methods}

To consider physiological loading conditions in vitro, the intradiscal pressure was first measured in 3 sheep over 24 hours in vivo. Average measured intradiscal pressure was $\sim 0.75 \mathrm{MPa}$ during day and $\sim 0.5 \mathrm{MPa}$ during night which corresponds to an axial force of 130 and $58 \mathrm{~N}$, respectively.

Two different loading protocols were performed in vitro: (1) an axial compressive stiffness test and (2) an axial compressive test of 3 loading cycles each consisting of 15 minutes at $130 \mathrm{~N}$ and 30 minutes at $58 \mathrm{~N}$. The tests were performed on 24 ovine motion segments $(16 \times$ L2-L3, $8 \times$ L4-L5).

Four different states were investigated: (i) INTACT, (ii) DEF-NUC: Nucleus tissue was removed and subsequently re-implanted and (iii-iv) two different hydrogels as nucleus replacements: DDAHA (Anika Therapeutics, Abano Therme, Italy) and iGG-MA (3 B's Research Group, University of Minho, Portugal).

During the test, the axial displacement and the nucleus pressure were recorded.

\section{Results}

In the stiffness test (1), the load-deformation-curves of all tested segments showed a nonlinear behavior characterized by a toe-region at the beginning of loading. This was followed by a progressive region and ended with an almost linear response.

$\rightarrow$ No significant differences were observed between the different testing groups.
In the compression test (2) INTACT showed a typical creep response with a steady loss of specimen height under the constant diurnal load of $130 \mathrm{~N}$. The night load only led to a slight recovery. Two intervals of night load did not compensate for the loss of height resulting from two intervals of diurnal load. Intact ovine discs caused a nucleus pressure of $\sim 0.3 \mathrm{MPa}$ without any external load. The application of 130 and $58 \mathrm{~N}$ initially caused the desired nucleus pressures of 0.75 and $0.5 \mathrm{MPa}$, respectively. During the diurnal load, the pressure slightly decreased by $\sim 3 \%$, while it remained almost constant during the night load.

$\rightarrow$ DEF-NUC, DDAHA and iGG-MA increased the height-loss (maximal for DEF-NUC: $~ 33 \%$ ) and decreased the fluid pressurization (maximal for DEF-NUC: $\sim 26 \%$ ) compared to INTACT.

\section{Discussion}

The re-implantation of the natural nucleus, assumed as being the ideal implant, was not able to restore the mechanical function of the disc. This may be due to (i) a damaged natural interface between nucleus and surrounding structures, (ii) an inadequate annulus sealant and (iii) a destroyed collagen-proteoglycan compound making up the native nucleus tissue. Therefore, hydrogels that mimic the mechanical behaviour of the native nucleus may still fail in restoring the disc height and fluid pressurization when neglecting the disturbed structural interrelations.

\section{Acknowledgements}

This work is funded by the EU project DISC REGENERATION (NMP-2007-CPIP213904), and by the German Research Foundation (WI 1352 14$1)$. 